\title{
Rehabilitación pulmonar en tuberculosis multirresistente (TB-MDR): informe de un caso
}

\author{
Esther Cecilia Wilches, FT'1 , Julián Andrés Rivera, FT², \\ Ricardo Mosquera, MD³ ${ }^{3}$, Liliana Loaiza, TO ${ }^{4}$, Lucely Obando, Psicol ${ }^{5}$
}

\section{RESUMEN}

Introducción: En la tuberculosis (TB) el gran componente inflamatorio, ocasiona lesiones importantes que desencadenan reacción fibroblástica, fibrosis y retracción de la pared costal, y comprometen la expansión pulmonar lo que se traduce clínica $\mathrm{y}$ funcionalmente en un patrón restrictivo moderado y disnea al ejercicio. Lo anterior favorece la discapacidad pulmonar, y ocasiona dependencia económica y social del núcleo familiar. Las medidas tendientes a controlar la enfermedad tuberculosa son sólo curativas, hecho que se debe considerar como insuficiente porque las acciones dirigidas hacia la habilitación y rehabilitación podrían evitar o disminuir la incidencia de discapacidad cardiopulmonar por TB. La importancia de la rehabilitación pulmonar (RP) como tratamiento no farmacológico en pacientes con enfermedades respiratorias crónicas y/o con factores de riesgo para adquirirlas, está documentada en la literatura, y su aplicación permite mejorar la condición física del paciente, restaurar la calidad de vida relacionada con la salud (CVRS), la autonomía y la integración social.

Objetivo: Describir el deterioro de la funcionalidad de un paciente con tuberculosis multirresistente (TB-MDR) y su proceso de recuperación en un programa de RP.

Resultados: Se observó un aumento en la distancia recorrida en el test de caminata de los seis minutos (TC6M) de 240 ma 350 m. La puntuación de disnea con la escala del Medical Research Council (MRC) mejoró de 4 a 1 y con la escala de Borg mejoró de 7 a 0 . La fuerza en la musculatura de los miembros superiores e inferiores aumentó de 3 a 4 .

Conclusión: Para este paciente un periodo de RP entre 8 y 10 semanas de duración, fue suficiente para mejorar la funcionalidad.

Palabras clave: Rehabilitación pulmonar; Tuberculosis multirresistente; Funcionalidad.

\section{Pulmonary rehabilitation in multi-drug resistant tuberculosis (TB MDR): a case report}

\section{SUMMARY}

Introduction: In tuberculosis (Tb), the great inflammatory component causes major injuries that trigger fibroblastic reaction, fibrosis and chest wall retraction, compromising pulmonary expansion, which translates into a clinically and functionally moderate restrictive pattern and dyspnea during exercise. This favors lung disability, causing economic and social dependence upon the nuclear family. Measures to control the $\mathrm{Tb}$ disease are merely focused on healing; and this fact must be considered insufficient because the actions aimed to habilitation and rehabilitation could prevent or reduce the incidence of Tb by cardiopulmonary disability. The importance of pulmonary rehabilitation (PR) as a nonpharmacological treatment in patients with chronic respiratory disease and/or risk factors for acquiring such is documented in the literature, and its application improves the physical condition of the patient and restores health-related quality of life (HRQOL), autonomy, and social integration.

1. Profesora Asistente, Programa de Fisioterapia, Directora Grupo de Investigación, Ejercicio y Salud Cardiopulmonar, Facultad de Salud, Universidad del Valle, Cali, Colombia. e-mail: esterwil@univalle.edu.co

2. Fisioterapeuta Asistencial, Programa de Rehabilitación Pulmonar, Hospital Universitario del Valle, Cali, Colombia. e-mail:juliancho2112@hotmail.com

3. Médico Neumólogo, Unidad de Medicina Interna, Hospital Universitario del Valle, Cali, Colombia. e-mail:rpmosquera@hotmail.com

4. Terapeuta Ocupacional, Unidad de Medicina Física y Rehabilitación, Hospital Universitario del Valle, Cali, Colombia. e-mail: lilo69gallardo@yahoo.com.ar

5. Psicóloga, Unidad de Quemados, Hospital Universitario del Valle, Cali, Colombia. e-mail: lucelyo@gmail.com Received for publication June 3, 2008 Accepted for publication September 30, 2009 
Objective: To describe the deterioration of the functionality of a patient with multi-drug resistant tuberculosis (MDR-TB) and that patient's recovery in a PR program.

Results: An increase of the distance covered in the sixminute walk test (6MWT) from $240 \mathrm{~m}$ to $350 \mathrm{~m}$ was observed. Dyspnea score with the medical research council (MRC) improved from 4 to 1 , and improved from 7 to 0 with the Borg scale. The upper and lower limb muscle strength increased from 3 to 4 .

Conclusion: A period of PR of 8 to 10 weeks was enough to improve patient functionality.

\section{Keywords: Pulmonary rehabilitation; Multi-drug resistant tuberculosis; Functionality.}

La multirresistencia a drogas antituberculosas (definida como la resistencia, por lo menos, a isoniazida y rifampicina) se acepta ampliamente como una amenaza para el control de la tuberculosis en varios lugares del mundo.

En Colombia, según la OMS/OPS (2004) el cálculo porcentual de tuberculosis multirresistente en enfermos con tuberculosis sin tratamiento previo estaba alrededor de $1 \%$ a $3 \%$, sin embargo, no se encuentran datos del porcentaje estimado de tuberculosis multiresistente en pacientes con tuberculosis previamente tratada ${ }^{1}$. Para el año, 2005 se diagnosticaron cerca de 10,000 casos de Tb y aproximadamente $15 \%$ de ellos se descubrieron en el Valle del Cauca (Guía Nacional de TB, 2005).

Ante enfermedades pulmonares como la $\mathrm{Tb}$ que no se han podido prevenir y mucho menos curar, la rehabilitación surge como la mejor posibilidad dirigida a disminuir su impacto sobre las vidas de quienes la sufren y a disminuir también sus consecuencias sociales y económicas. La evidencia científica acerca de los beneficios de los programas de rehabilitación pulmonar es sólida, y se han comprobado en todo sujeto con enfermedad respiratoria crónica y disnea, independientemente del grado de severidad de la enfermedad, tanto a nivel de hospitales como ambulatorio y en la casa. El paciente con deterioro pulmonar crónico presenta aumento del trabajo respiratorio, alteración muscular, depresión y cambios nutricionales que forman un círculo vicioso de inactividad y mayor desgaste fisiológico que incluso se puede presentar en reposo; este ciclo se debe intervenir con un manejo integral adecuado en un programa de rehabilitación pulmonar que contemple un mínimo de seis semanas de ejercicio físico, (nivel de evidencia B), entrenamiento físico aeróbico obligatorio, caminata o cicloergómetro, con nivel de evidencia (A), educación al paciente y a la familia (evidencia 1B), intervención psicosocial (evidencia 2B) y nutrición (evidencia 2C) ${ }^{2}$.

Este artículo describe la decadencia en la funcionalidad respiratoria de un enfermo con tuberculosis multirresistente (TB-MDR) que llegó hasta la pérdida de su capacidad para cumplir las actividades de la vida diaria y su evolución en un programa de rehabilitación pulmonar. En el medio colombiano son escasas las comunicaciones encontradas al respecto.

\section{INFORME DEL CASO}

Se describen los componentes que se siguieron en un programa de rehabilitación pulmonar para un paciente con TB-MDR, según el marco de referencia de La Guía de Práctica Clínica de Fisioterapia publicada por la Asociación Americana de Terapia Física (examen, evaluación, diagnóstico, intervención, re-evaluación), como también, las definiciones conceptuales sobre las cuales se fundamenta el modelo de la Clasificación Internacional del Funcionamiento y Discapacidad(CIF) deficiencia, limitación de la actividad y restricción en la participación: Paciente de 26 años, sexo masculino, raza negra, bachiller, procedente de Buenaventura (Colombia), puerto sobre la costa colombiana del Pacífico, con alta incidencia de tuberculosis, donde $48 \%$ de la población tiene sus necesidades básicas insatisfechas; el desempleo estimado es de $60 \%$ y el aseguramiento en salud es inferior a 40\%) ${ }^{1}$. Paciente sin antecedentes de tabaquismo, sedentarismo y/o exposición a sustancias tóxicas, nivel de educación básico primaria-secundaria y estrato socioeconómico bajo.

Al paciente se le diagnosticó Tb pulmonar en 1997; presentaba historia de tratamiento con múltiples esquemas. Al comienzo recibió isoniazida, rifampicina, pirazinamida y etambutol, medicamentos que suspende para someterse a manejo casero a base de hierbas recomendado por curandero de la comunidad. Su situación, en el período de diciembre 2004 a 2006 se desconoce, hasta que ingresa al Hospital Universitario del Valle, en julio de 2006, con insuficiencia respiratoria.

Ingresa al Programa de Rehabilitación Pulmonar del Hospital Universitario del Valle (en diciembre de 2007), desplazándose en silla de ruedas, que maneja el cuidador, de quien depende entre $10 \%$ y $100 \%$ para las 
actividades de la vida diaria (AVD) y en las actividades instrumentales es por completo dependiente.

En la evaluación fisioterapéutica inicial se identificaron deficiencias en la capacidad aeróbica, en la ventilación e intercambio gaseoso, en la movilidad articular y en el desempeño muscular. Los campos pulmonares con murmullo vesicular disminuido globalmente con roncos, estertores y sibilancias difusas; el enfermo dependía por completo de oxígeno, con cánula nasal a $4 \mathrm{l} / \mathrm{m}$ de manera continua, habla entrecortada, patrón respiratorio rápido, superficial de predominio costal superior, con disnea a esfuerzos pequeños, expansibilidad torácica disminuida en ambas bases; tos efectiva con expectoración de secreciones mucoides en moderada cantidad, con pérdida de fuerza muscular generalizada en miembros superiores e inferiores según la escala de Daniels (3/5). En la prueba de función pulmonar la curva de flujo volumen mostró severo compromiso mixto (VEF1 15\%, CVF 31\%, VEF1/CVF 40\%) sin respuesta a broncodilatador inhalado; los gases arteriales mostraron hipoxemia. La tomografía computadorizada de tórax mostró patrón de vidrio esmerilado en ápices pulmonares, bulas enfisematosas, cavernas en el ápex derecho y bronquiectasia bilateral.

En la prueba de (test) caminata de los seis minutos (6MWT), realizada en un pasillo de 30 metros se registraron la saturación parcial de oxígeno $\left(\mathrm{SpO}_{2}\right)$ y la frecuencia cardíaca (FC) durante toda la prueba, monitorizada a través de la telemetría inalámbrica. Al principio y al final de la prueba se evaluó el grado de disnea y el grado de fatiga con la escala modificada de Borg. Al finalizar se registraron las vueltas y los metros recorridos. Se hicieron dos pruebas en horas de la mañana y se registró la de mayor valor en metros. Se le ajustó el flujo de oxígeno, para mantener una $\mathrm{SpO}_{2}$ $\geq 90 \%$. Al iniciar y finalizar la rehabilitación, la disnea crónica durante las AVD se cuantificó con la escala Medical Research Council (MRC).

El paciente presentaba deterioro en la calidad de vida, por aumento de síntomas respiratorios que le limitaban las AVD como trabajar, estudiar, salir a visitar a familiares y amigos y restringía su participación en actividades sociales.

En la evaluación inicial del desempeño en el área de las AVD (auto cuidado, higiene y vestido, alimentación) el paciente logró una participación de $10 \%$ a $100 \%$ y en las instrumentales (preparación de alimentos, manteni- miento del hogar, cuidado de otros) era dependiente por entero del cuidador. No hacía actividades de tiempo libre/ocio ni productivas. En cuanto al estado emocional, en la valoración inicial por medio de entrevista clínica se encontró un individuo consciente, orientado en las tres esferas, tiempo, persona y lugar, con una percepción de símismo como «inútil», con pensamientos de minusvalía, poca tolerancia a la frustración y con un reconocimiento de la responsabilidad directa que tuvo sobre su actual estado físico. Se aplicó la escala Depresión y Ansiedad en el Hospital (Hospital Anxiety and Depression Scale HAD) con puntaje de dos, en el componente de depresión, y siete en el componente de ansiedad, en relación con miedos y temores ocasionados por su problema de salud.

En este caso en particular, hubo dificultades administrativas para una valoración nutricional completa y el paciente sólo recibió algunas recomendaciones dietéticas.

El componente educativo en este caso no se evaluó ni se desarrolló por entero, sin embargo, en las sesiones de entrenamiento, el contenido educativo se adaptó a su situación social y cultural, se brindó información acerca de la enfermedad y asesoramiento en el uso de inhaladores, en los métodos para mejorar la disnea y se brindaron pautas para el manejo y prevención de las exacerbaciones.

La calidad de vida se evaluó al inicio y al final del programa de entrenamiento mediante el cuestionario de salud SF-36 v.2, que tiene un rango de 0 a 100 y los puntajes más altos indican una mejor calidad de vida. Se solicitó el permiso para usar el cuestionario a la Unidad de Investigación en Servicios Sanitarios (IMIM) de España.

Después de la valoración inicial por todo el equipo interdisciplinario, se estableció un programa de intervención que constaba de entrenamiento de fuerza y resistencia de miembros inferiores, miembros superiores, educación y técnicas de conservación de energía. En este caso, el entrenamiento físico se hizo en bicicleta ergométrica, banda sin fin y step, empleando al principio una carga equivalente a $30 \%$ de la carga máxima, que se aumentó de modo progresivo hasta alcanzar un máximo preestablecido de $85 \%$, o en su defecto, la carga máxima que el paciente pudiera sostener durante $60 \mathrm{~min}$. Todas las sesiones de entrenamiento las supervisó un fisioterapeuta especialista en rehabilitación car- 
díaca y pulmonar. Se trabajó entrenamiento de fuerza y resistencia de miembros superiores e inferiores. Durante los ejercicios, se ajustó el flujo de oxígeno para mantener una saturación parcial de oxígeno $\left(\mathrm{SpO}_{2}\right)$ superior a $89 \%$ y se hizo broncodilatación previa al inicio de la actividad física. Asistió a rehabilitación tres veces por semana durante una hora, por un período de treinta y dos semanas, durante cuatro meses (hubo inconvenientes en las autorizaciones para la rehabilitación, recursos económicos y de transporte). Durante las sesiones de entrenamiento se observó un paciente colaborador, con expectativas, motivado, participativo en las actividades de educación y recreación, con permanente presencia y acompañamiento de los familiares.

El paciente firmó el consentimiento informado del Programa de Rehabilitación y también firmó la autorización para publicar este material.

\section{DISCUSIÓN}

La falla terapéutica en la $\mathrm{Tb}$ tiene repercusión en el ámbito laboral y social por la pérdida económica atribuida a la ausencia e incapacidad para trabajar, además de convertirse en un problema de salud pública porque el enfermo continúa como un foco infeccioso en la comunidad y puede conducir a la diseminación de formas resistentes. Los factores de riesgo para el desarrollo de resistencia incluyen habitar en zona endémica de $\mathrm{Tb}$ con alta resistencia, contacto cercano y prolongado con personas infectadas con $\mathrm{Tb}$ resistente y terapia previa no curativa. Los daños residuales en el tejido pulmonar después de concluida la terapia antimicrobiana pueden incluir diversos grados de fibrosis, distorsión broncovascular, enfisema, bronquiectasias y aumento en la producción de esputo ${ }^{3}$.

Los pacientes pulmonares crónicos, pese a recibir todos los recursos farmacológicos disponibles, continúan por lo general con limitación física al esfuerzo. En la $\mathrm{Tb}$ el gran componente inflamatorio, ocasiona lesiones importantes que desencadenan reacción fibroblástica, fibrosis, retracción de la pared costal, y afectan la movilidad, lo que complica la expansión pulmonar. La función del diafragma se altera, las adherencias pleurodiafragmáticas lo retraen y acortan y arriesgan la excursión normal. Estas perturbaciones mecánicas afectan a la mecánica ventilatoria a nivel costal y diafragmático. Lo anterior favorece la discapacidad pulmonar y origina dependencia económica y social del núcleo familiar. Las medidas que tienden a controlar la enfermedad tuberculosa son sólo curativas, hecho que se debe tener como insuficiente porque las acciones de habilitación y rehabilitación podrían evitar o disminuir la incidencia de discapacidad cardiopulmonar por $\mathrm{Tb}$. Las personas que han completado tratamiento antituberculoso evolucionan con discapacidad pulmonar debido a la enfermedad fibrocavitaria.

Algunos estudios muestran los beneficios de un programa de rehabilitación pulmonar en pacientes con historia de tuberculosis.

Ando et al. ${ }^{4}$ compararon los efectos de un programa de rehabilitación de nueve semanas de intervención en individuos con EPOC y en sujetos con secuelas de Tb y encontraron que no hubo cambios significativos en el VEF1 después de la rehabilitación en ambos grupos, pero si evidenciaron mejoría en la disnea medida con la MRC en las AVD y en la distancia recorrida en la prueba de caminata $42 \mathrm{~m}$ en pacientes con $\mathrm{Tb}$ y $47 \mathrm{~m}$ en pacientes con EPOC $(\mathrm{p}<0.01)$ datos que concuerdan con este informe donde la distancia recorrida en $6 \mathrm{~min}$ aumentó de $240 \mathrm{~m}$ a $350 \mathrm{~m}(110 \mathrm{~m})$, pese a que no alcanzó el predicho de $740 \mathrm{~m}$ calculado con la fórmula de Enrigth. La frecuencia respiratoria y la frecuencia cardíaca disminuyeron al finalizar el programa de rehabilitación (Gráfica 1). Al determinar la magnitud de los cambios y las características en este paciente, luego del programa de rehabilitación pulmonar, se encontró que hubo mejoría en la percepción de la disnea medida con la escala de la MRC, de 4 a 1 (4: muy grave; 1: leve) y con la escala de Borg mejoró de 7 a 0 . La necesidad de oxígeno se re-evaluó al finalizar la rehabilitación y se observó mejoría, pues pudo permanecer en estado de reposo sin soporte de oxigeno manteniendo $\mathrm{SpO}_{2}$ mayor de $89 \%$ y siendo posible la disminución de la $\mathrm{FIO}_{2}$ de 5 a 1 litros/m durante las AVD. La fuerza global en la musculatura de los miembros superiores e inferiores aumentó de grado 3 a 4, según la escala de Daniels, "ue va desde el grado 0 al 5 ( 3 el movimiento puede vencer la acción a la gravedad y grado 4 movimiento en contra de la gravedad con resistencia parcial).

Pasipanoyda et al. ${ }^{5}$, validaron el cuestionario de calidad de vida St George (SGRQ) en una población microbiológicamente curada de $\mathrm{Tb}$ y encontraron una diferencia (media de 13.5 - U) en el puntaje del SGRQ entre estos enfermos y un grupo control ( $\mathrm{Tb}$ latente) con 


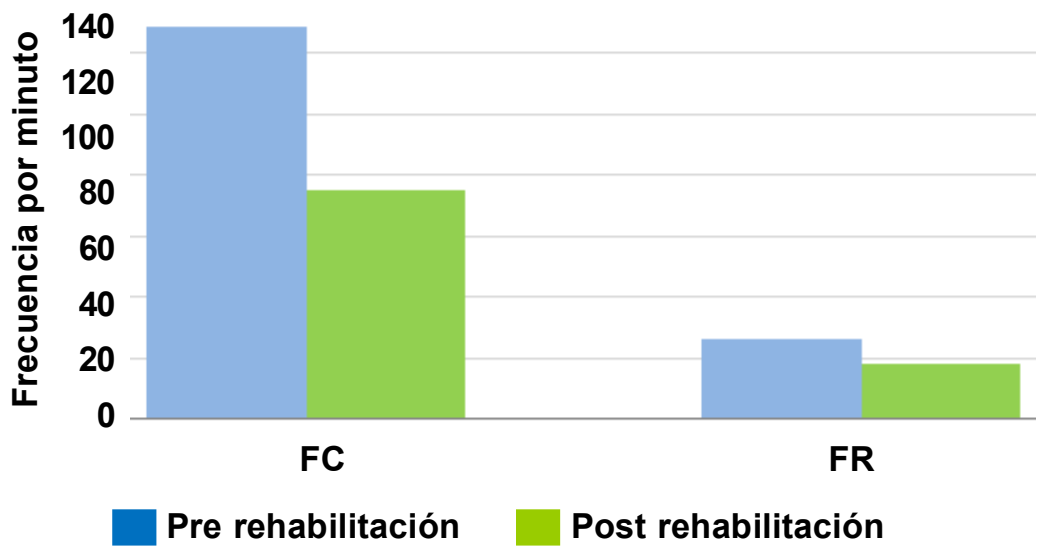

Gráfica 1. Signos vitales pre y post rehabilitación

FC: Frecuencia cardíaca; FR: Frecuencia respiratoria. En color azul los valores alcanzados pre-rehabilitación y en verde los valores post-rehabilitación. Julio 24 de 2007. Programa de Rehabilitación Pulmonar.

factores de riesgo similares $(\mathrm{p}<0.001)$. La media total del puntaje para los tuberculosos tratados tuvo significancia más alta que para los sujetos con Tb latente (23.5 vs. 10.3), respectivamente $\mathrm{p}<0.001$. Los investigadores sugieren que esta diferencia demuestra el deterioro después del tratamiento antituberculoso, lo que tiene gran impacto sobre la salud a nivel mundial y concluyeron que la cura microbiológica de la $\mathrm{Tb}$ no es suficiente para evitar la pérdida de salud.

En este paciente, la calidad de vida se midió con el SF-36v2, y con respecto a las dimensiones físicas (función física, rol físico, dolor corporal, vitalidad y salud general) y las dimensiones psicosociales (función social, salud mental y rol emocional) que conforman el cuestionario, se encontraron valores medios y altos al final del programa de entrenamiento, lo que indica a nivel general una calidad de vida buena en estas dimensiones, cuando se compararon con las puntuaciones obtenidas en la evaluación inicial, como se muestra en la Gráfica 2.

Al finalizar el programa de rehabilitación el paciente logró funcionalidad en el desplazamiento en superficies planas, subir y bajar escaleras; su participación en las actividades de autocuidado mejoró hasta alcanzar 100\% de independencia y mejoró su participación en las actividades de ocio y tiempo libre. Actualmente presenta expectativas de iniciar capacitación en sistemas de informática para complementar sus áreas productivas. La hipoacusia se manejó con audífonos, y esto permitió mejorar su función comunicativa.

En la re-evaluación psicológica se encontró un paciente con percepción positiva de sí mismo, evidenciado en su discurso, refiriéndose a su estado actual de salud como «muy bueno», con puntuación en la escala HAD en cero (0) para ambos estados. Se observó en este caso un claro deseo de mejorar aún más y seguir creciendo en su vida, tanto personal como profesional.

La respuesta satisfactoria al programa de rehabilitación pulmonar en términos de funcionalidad, animó a los autores a comunicar los resultados obtenidos, que aunque no se pueden extrapolar a toda la población con TBMDR, pueden ser iniciadores en la fundamentación de los procesos de rehabilitación en estos casos. Para este enfermo con TB-MDR, un período de rehabilitación pulmonar de 32 sesiones, fue suficiente para mejorar la distancia recorrida en el (TC6M), mejorar las AVD, mejorar la calidad de vida y disminuir síntomas.

La educación al paciente y su familia que se considera como un componente esencial en la atención de cualquier enfermedad crónica, en este caso fue un componente poco desarrollado, al igual que el componente nutricional, por lo que, se recomienda establecer en el programa de rehabilitación estrategias que faciliten el desarrollo del factor educativo y que aseguren la evaluación y acompañamiento del estado nutricional de los pacientes.

En este tipo de pacientes se necesitan estudios con 


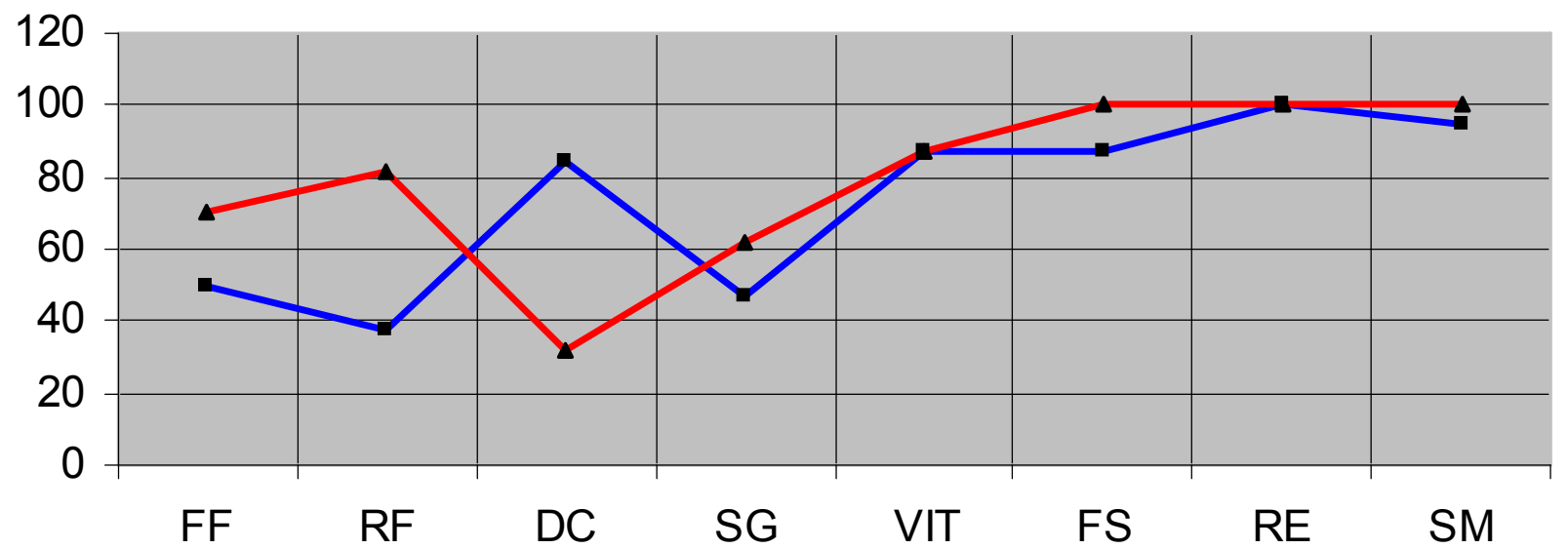

Gráfica 2. Resultados SF-36 v.2

La línea de color azul hace referencia a los resultados en el pre-test. La línea roja hace referencia a los valores en el post-test. FF: Función física: RF: Rol físico; DC: Dolor corporal; SG: Salud general; VIT: Vitalidad; FS: Funcionamiento social; RE: Rol emocional; SM: Salud mental

Julio 24 de 2007. Programa de Rehabilitación Pulmonar

otras medidas de evaluación de resultados, como el número dehospitalizaciones y participación en las AVD, para confirmar los resultados obtenidos. Aunque la experiencia en este tema es limitada, conviene pensar no sólo en adecuar las estrategias curativas para los pacientes con TB MDR sino también en el proceso de rehabilitación e integrar un conjunto de acciones con el objetivo de lograr que los enfermos con discapacidad cardiopulmonar alcancen un nivel funcional, mental y/o social óptimo, en los distintos ámbitos donde se desempeñen en la vida cotidiana.

\section{REFERENCIAS}

1. Asociación Latinoamericana de Tórax(ALAT). Guías latinoamericanas de diagnóstico y tratamiento de la tuberculosis fármaco resistente. [cited 2007 Abr 7]. Available from: URL: http://www.alatorax.org/archivos/guias TBCfinales.pdf

2. Charles F, Ries AL, Bauldoff S, Carlin BW, Casaburi R, ZuWallack R, et al. Pulmonary rehabilitation: ACCP/AACVPR evidence-based clinical: Practice guidelines. Chest. 2007; 131: 4-42.

3. Hnizdo E, Singh T, Churchyard G. Chronic pulmonary function impairment caused by initial and recurrent pulmonary tuberculosis following treatment. Thorax. 2000; 55:32-8.

4. Ando M, Mori A, Esaki H, Shiraki T. The effect of pulmonary rehabilitation in patients with post-tuberculosis. Lung Disorder Chest. 2003; 123:1988-95.

5. Pasipanodya JG, Miller TL, Vecino M, Munguia G, Bae S, Drewyer G. Using the St. George Respiratory Questionnaire to ascertain health quality in persons with treated pulmonary tuberculosis. Chest. 2007; 132: 1591-8. 